\title{
Partnership models for the establishment of sustainable paediatric cardiac surgical and cardiac intensive care programmes in low- and middle-income countries
}

\author{
Patricia Bastero, ${ }^{1}$ Sandra L. Staveski, ${ }^{2}$ Bistra Zheleva, ${ }^{3}$ Emma Scanlan, ${ }^{4}$ Antonio G. Cabrera, ${ }^{1}$ Aric Araujo, ${ }^{5}$ \\ Guillermo Reyes, ${ }^{1}$ Carlos M. Mery, ${ }^{6}$ Alexis Palacios-Macedo, ${ }^{7}$ Christian P. Brizard ${ }^{8}$ \\ ${ }^{1}$ Cardiac Intensive Care Unit, Texas Children's Hospital, Baylor College of Medicine, Houston, Texas; ${ }^{2}$ Cincinnati Children's \\ Hospital Medical Center, Cincinnati, Obio; ${ }^{3}$ Children's HeartLink, Minneapolis, Minnesota, United States of America; \\ ${ }^{4}$ Chain of Hope, London, United Kingdom; ${ }^{5}$ Cardiac Intensive Care Unit, Instituto Nacional de Pediatria Hospital and ABC \\ Hospital, Mexico City, Mexico; ${ }^{6}$ Congenital Heart Surgery, Texas Children's Hospital, Baylor College of Medicine, Houston, \\ Texas, United States of America; ${ }^{7}$ Cardiovascular Surgery, Instituto Nacional de Pediatria Hospital and ABC Hospital, \\ Mexico City, Mexico; ${ }^{8}$ Cardiac Surgery Department, The Royal Children's Hospital Melbourne, Parkville, Victoria, Australia
}

\begin{abstract}
The care of patients with CHD remains a challenge in low- and middle-income countries. Their health systems have not been able to achieve consistently high performance in this field. The large volume of patients, manpower constraints, inconsistencies in the level and type of background training of the teams caring for this patient population, and the inadequate quality control systems are some of the barriers to achieving excellence of care. We describe three different international projects supporting the paediatric cardiac surgical and paediatric cardiac intensive care programmes in Latin America, Asia, and the Caribbean.
\end{abstract}

Keywords: Low- and middle-income countries; paediatric cardiac intensive care; sustainability

Received: 15 September 2017; Accepted: 15 September 2017

$\mathrm{T}$ HE WORLD'S CURRENT POPULATION IS $\sim 7.2$ billion. While most of the population lives in Asia, the highest rate of population increase is in Africa. These countries are affected by the "double disease burden": communicable or infectious diseases and non-communicable diseases. It is estimated that non-communicable diseases contribute up to a third of the total disease burden, and they are estimated to reach up to $45 \%$ by the year 2030 . Noncommunicable disease-related mortality is estimated to cause as many as $80 \%$ of all deaths in low- and middle-income countries. CHD is one of the most common non-communicable diseases, with an incidence that ranges from 1.2 to 17 per 1000 live births. ${ }^{1}$

Correspondence to: P. Bastero, MD, Cardiac Intensive Care Unit, Texas Children's Hospital, Baylor College of Medicine, 6621 Fannin St., Houston, TX 77030, United States of America. Tel: (+1) 832-8266230; Fax: (+1) 832-8257422; E-mail: pxbaster@texaschildrens.org
Despite over 300 million patients undergoing different surgical procedures worldwide every year, there is epidemiological data suggesting that over another 140 million are needed, mostly in low- and middle-income countries. The reality is that, in those countries, over 4.5 million people do not have access to safe surgical treatment. ${ }^{2}$ About $90 \%$ of those children have access only to suboptimal care, if they have any access to treatment at all. ${ }^{3}$ Some children also require surgical procedures for acquired cardiac disease - e.g., rheumatic fever.

Surgical accessibility is not the only problem for children in low- and middle-income countries. They are at risk of high morbidity and mortality rates postoperatively, with some reports showing mortality rates 10 times higher in low- and middle-income countries when compared with high-income countries. ${ }^{4}$ Safe perioperative practices, reduction of infections, and team-based practices are a few of the 
reported successful factors in the optimisation of the care of children with CHD in low- and middleincome countries. ${ }^{4}$

The objective of this paper is to present four different models of international partnership programmes in Mexico, India, Vietnam, and Jamaica.

\section{Mexico}

There are $\sim 18,000$ children diagnosed with CHD each year in Mexico. It is estimated that only a third of those children receive access to treatment, and frequently this treatment is suboptimal. Public hospitals have a large volume of patients, but inadequate quality control systems and limited resources. Private hospitals have the resources but a low volume of patients.

A local team in Mexico led by the head cardiovascular surgeon at Instituto Nacional de Pediatria helped create the Kardias Foundation. Kardias raises money through donations and invests it in the support of all aspects involved in the care of children with $\mathrm{CHD}$, including diagnosis, referral for surgical treatment, and family support. After establishing the collaboration between Instituto Nacional de Pediatria and Kardias, the mortality rate of CHD decreased by $14 \%$. However, it was still far higher than in high-income countries. Kardias and Instituto Nacional de Pediatria then partnered with a private institution, the $\mathrm{ABC}$ hospital. They built a hybrid system using elements from both public and private sectors to create a centre of excellence for the care of children with $\mathrm{CHD}$, combining the high volume of patients attended to at public hospitals with the resources and high quality controls of a private hospital certified by the Joint Commission on Accreditation of Health Care Organizations. They also received the support from the Mexican government. Along with this partnership, a "twinning" project with Texas Children's Hospital was signed. Texas Children's Hospital provides needs assessment, educational activities, interdisciplinary communication optimisation efforts, weekly cardiac intensive care case reviews, and surgical planning meetings using telemedicine, surgical visits to $A B C$ hospital, observer-ships at Texas Children's Hospital, and "as needed" 24/7 consulting via several different media technologies.

The prioritised needs assessment outcomes related to team performance identified in the cardiac ICUs after several visits to Instituto Nacional de Pediatria and ABC hospitals by a multidisciplinary team from Texas Children's Hospital, and the actions taken by the group to improve them, are shown in Table 1 . The focus of all educational activities conducted by Texas Children's Hospital was on the training of nurses in the basics of CHD management. The focus of simulation-based activities was the reinforcement
Table 1. Needs assessment and actions taken for improvement in the ICU setting; based on the ABC Hospital Mexico City and Texas Children's Hospital example presented.

\begin{tabular}{|c|c|}
\hline $\begin{array}{l}\text { Identified point for } \\
\text { improvement }\end{array}$ & Action taken \\
\hline OR to ICU sign out & $\begin{array}{l}\text { Scripted organised sign out } \\
\text { between teams }\end{array}$ \\
\hline $\begin{array}{l}\text { Lack of nursing experience with } \\
\text { CHD patients }\end{array}$ & $\begin{array}{l}\text { Didactic lectures and simulation- } \\
\text { based activities on CICU topics }\end{array}$ \\
\hline $\begin{array}{l}\text { Poor multidisciplinary team } \\
\text { adherence }\end{array}$ & $\begin{array}{l}\text { Simulation-based } \\
\text { multidisciplinary team training }\end{array}$ \\
\hline $\begin{array}{l}\text { High incidence of central-line- } \\
\text { associated infections }\end{array}$ & $\begin{array}{l}\text { Skill stations for safe central line } \\
\text { management and bedside } \\
\text { nursing training at } \mathrm{TCH}\end{array}$ \\
\hline $\begin{array}{l}\text { Lack of preparation for high- } \\
\text { acuity low-incidence events } \\
\text { such as cardiac arrest }\end{array}$ & Regular mock codes in the unit \\
\hline $\begin{array}{l}\text { Excess time to access critical } \\
\text { medications and equipment }\end{array}$ & Crash cart inside the $\mathrm{CICU}$ \\
\hline $\begin{array}{l}\text { Lack of preparation for high- } \\
\text { acuity low-incidence events } \\
\text { such as cardiac arrest }\end{array}$ & $\begin{array}{l}\text { Documentation of pre-calculated } \\
\text { critical medication doses at } \\
\text { patient's bedside }\end{array}$ \\
\hline $\begin{array}{l}\text { Lack of standardisation in the } \\
\text { preparation of medication } \\
\text { drips }\end{array}$ & $\begin{array}{l}\text { Consistent drip (concentration) } \\
\text { preparation }\end{array}$ \\
\hline
\end{tabular}

of the cognitive skills taught during didactic lectures and seminars, and the building of a team, with the main objective of optimising interdisciplinary communication.

The specifics of weekly telemedicine activities held by Texas Children's Hospital and the local team in Mexico are presented in Figure 1. A total of 320 patients have been operated on at $\mathrm{ABC}$ hospital since the start of the collaboration and twining programmes. A total of 278 of those patients were derived from the public sector, and 42 patients from the private sector. During the first 17 months, only RACHS- 1 level 1 and two cases were performed, with zero mortality. As the programme evolved, the percentage of RACHS-1 level 3 to 6 and of Aristotle score 3 to 5 per year steadily increased. RACHS-1, Aristotle score adjusted, and STS-EACTS cases are presented in Table 2. Overall mortality is $2.81 \%$, comparable to that in high-income countries.

\section{India}

The burden of CHD in Asia is the largest in the world because of the large population and higher birth rates in this region. ${ }^{5}$ A significant challenge is providing highquality paediatric cardiac services, especially in critical care. Contributing factors include insufficient specialised centres that provide high-quality surgical and critical care, lack of academic training programmes, 


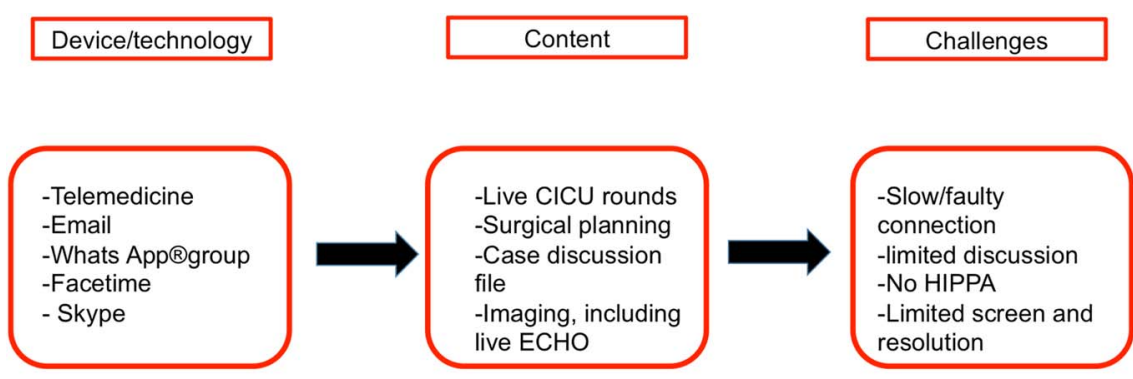

Telemedicine equipment can experience difficulties due to local technical expertise, equipment availability, variable internet speed connection or institutional firewall barriers. Particularly, dynamic angiographic images need higher internet speed or they will appear pixelated. WhatsApp is now encrypted and also has video streaming capabilities but data may need to be de-identified. Email is helpful when off line surgical opinion is needed.

Figure 1.

Weekly telemedicine activities' characteristics. CICU = cardiac ICU.

Table 2. Percentages of patients with RACHS-1, Aristotle adjusted score, and STS-EACTS, based on the ABC Hospital Mexico City and Texas Children's Hospital example presented.

\begin{tabular}{cc}
\hline & Percentage \\
\hline RACHS-1 & \\
1 & $32 \%(103)$ \\
2 & $45 \%(144)$ \\
3 & $17 \%(54)$ \\
4 & $5 \%(16)$ \\
5 & $0 \%(0 \%)$ \\
6 & $1 \%(2)$ \\
Aristotle adjusted & \\
1 & $32 \%(103)$ \\
2 & $31 \%(99)$ \\
3 & $20 \%(64)$ \\
4 & $16 \%(51)$ \\
5 & $1 \%(3)$ \\
STS-EACTS & \\
1 & $44 \%(142)$ \\
2 & $33 \%(106)$ \\
3 & $6 \%(20)$ \\
4 & $16 \%(50)$ \\
5 & $1 \%(2)$ \\
\hline
\end{tabular}

insufficient ancillary services such as physiotherapy and social workers, and the lack of acceptance that paediatric cardiac critical care needs to exist as a separate discipline. Many children in low- and middle-income countries still receive surgery and recover in adult ICU.

Amrita Institute of Medical Sciences in Kochi, India, performs 600 paediatric cardiac surgeries per year. Recently, the institute began offering a 2-year fellowship in paediatric cardiac intensive care with mandatory eligibility for completed speciality training in anaesthesia or paediatrics and desirable fellowship in cardiac anaesthesia or paediatric cardiology. To improve critical care nursing, Institut Jantung Negara (National Heart Institute) in Kuala Lumpur, Malaysia, started a 6-month specialised advanced nursing training programme creating nursing "mentors" in 2006 through a collaboration with Istitut Jantung Negara College and Liverpool John Moores University. The mentors facilitate training of nurses in the paediatric cardiac ICU. To address the difficulty of hiring well-trained intensivists, in 2013 Istitut Jantung Negara developed a 1-year specialised Clinical Site Nurse Practitioner course. The nurses graduating from the Clinical Site Nurse Practitioner course function in a similar role as an intern in the United States in order to supplement paediatric cardiac ICU staffing. The expanded role of mentors and Clinical Site Nurse Practitioners empowers nurses to optimise care for this vulnerable, complex patient population.

Another example of nursing empowerment is the Parent Education Discharge Instruction programme. The Parent Education Discharge Instruction programme was developed to educate bedside nurses on the importance of discharge teaching and to provide them with a structured process for conducting parent teaching for home care of children after cardiac surgery. ${ }^{6-8}$ The home care education intervention includes a sustainable electronic nursing training session and diverse parent home care education opportunities - e.g., individualised teaching, materials including pictograms for parents/caregivers with low literacy, and posters. ${ }^{6-8}$

Three investigations examined the Parent Education Discharge Instruction programme in Southern India. The first investigation generated preliminary data on the feasibility and acceptability of the nurseled structured discharge programme. The structured nurse-led parent discharge-teaching programme demonstrated feasibility, acceptability, utility, and sustainability in the cardiac unit in Southern India. ${ }^{6}$ The next investigation evaluated the effectiveness of a structured nurse-led parent discharge-teaching programme on nurse, parent, and child outcomes. All three improved after implementation of the 
programme. ${ }^{7}$ This programme may help prepare parents to provide better home care for their children after cardiac surgery. Staveski et al found that parents had less overall uncertainty after implementation of the programme $(\mathrm{p}=0.001)$ and that nurses' and parents' perception of readiness for hospital discharge were in closer alignment after adoption of the programme. ${ }^{8}$ The programme has been implemented in at least 10 hospitals in five countries and reaches over 10,000 children and their families yearly. Each organisation has embraced the programme and adapted it to its local environment to ensure its success.

Another solution to this challenging situation has been capacity building support from overseas nongovernmental organisations. Children's HeartLink engages in long-term partnerships with hospitals from low- and middle-income countries to help them improve by developing high-quality fully integrated paediatric cardiac services through targeted capacity building activities and technical assistance, especially in the postoperative period. Intensive care is always a focus area for the training and mentoring activities and the ultimate aim of improving clinical outcomes. ${ }^{9}$ The organisation also takes advantage of its partners' participation in the International Quality Improvement Collaborative for Congenital Heart Surgery in Developing World Countries. This collaborative offers a free patient registry, benchmarked reporting, and monthly webinars on topics related to team-based practice, infection prevention, and safe perioperative practices. One International Quality Improvement Collaborative site reported improvements in key outcome measures such as bacterial sepsis (from 15.1 to $9.6 \%, \mathrm{p}<0.001$ ), surgical site infection (from 11.1 to $2.4 \%, \mathrm{p}<0.001$ ), and duration of ICU stay [from 114 (8-999) hours to 72 (18-999) hours $(\mathrm{p}<0.001)] .{ }^{10}$ The collaborative as a whole also reported improvement in infection rates. ${ }^{11}$

\section{Vietnam}

The Heart Institute "Viện Tim”, Ho Chi Minh City, was created in 1990 from the combined initiative from Pr Dương Quang Trung of Sở Y Tế (the Department of Health of Ho Chi Minh City) and Professor Alain Carpentier. This original structure is an independent non-profit Cardiac Surgery Hospital aimed at treating children. It is organised to be financially sustainable and autonomous. The Carpentier foundation and other charities provide partial or complete financial assistance to families so they may pay for the fixed price of open-heart surgery $\$ 2700$ USD in 2017.

Almost no paediatric cardiac surgery was performed in any part of Vietnam until the time the centre opened in 1992. Cardiologists, surgeons, nurses, and administrators had been trained in Paris during the construction of the hospital. For the first few years, French surgeons, cardiologists, intensivists, and nurses rotated from Paris to assist the local clinicians. Just over 400 patients were operated on in 1992.

In 2015, the facilities were replaced with a new building. Over the past 2 years, over 1600 open-heart surgeries were performed annually. The hospital has trained the vast majority of the cardiac surgeons and a significant proportion of the intensive care nurses now working in Vietnam. Since the beginning of the programme, in-hospital mortality has been below $2.5 \%$.

In 2003, the Royal Children's Hospital, Melbourne, Australia, was contracted by the charity Atlantic Philanthropies of Chuck Feeney to lead the build-up and training of the Heart Centre teams in the Central Hospital of Hué, Vietnam. A USD 5 million budget was devoted to the project, which covered the training of both adult and paediatric cardiologists, cardiac surgeons, intensivists and anaesthetists, nurses, and perfusionists. Supervised from Melbourne, the project involved the collaboration of several training centres: the Royal North Shore Hospital in Sydney for adult training, the Children's Hospital in Melbourne for the paediatric training, the Heart Institute in Ho Chi Minh City for the nurses training, and the Ecole National de Santé in Rennes, France, for the administrative staff. It was preceded by an accelerated language course in Hué for selected personnel. Follow-up missions from Sydney and Melbourne occurred to evaluate the training and provide further teaching. Currently, the Heart Centre in Hué performs over 800 open-heart surgeries per year, including neonatal and infant surgery.

From this extensive experience in Vietnam, some considerations pertinent to establishing sustainable paediatric cardiac surgical and cardiac intensive care programmes in low- and middle-income countries can be highlighted. Well-established programmes in high-income countries have grown slowly over several decades. Their strength is based on their medical and nursing teams, which have grown gradually in size, experience, and expertise. Establishing a sustainable programme in a low- and middle-income country is an attempt at accelerating this process, but only so much can be bypassed. It is essential to set achievable goals. Unfortunately, this generally means selecting a target group of patients or at least setting limits - e.g., no extracorporeal life support, no operating on hypoplastic-left heart syndrome, and so on. This will determine the following:

- The people who are going to work on this project: issues to consider include recruitment, training, maintaining motivation, financial remuneration, and teaching them how to train their successors. 
- The equipment acquired: knowing that the most up-to-date and sophisticated equipment may not be suitable or required for the degree of complexity targeted. A small upgrade in safety may mean a considerable increase in cost.

- A sustainable financial model to be implemented and monitored.

There are also specific medical issues relevant to low- and middle-income countries that add to the complexity of the undertaking, including the following: late presentation $\mathrm{CHD}$; access to prostaglandin; infection issues, such as availability of antimicrobials and microbiology laboratories, and the impact of neglected tropical diseases on patient care; malnutrition; and adequate blood bank facilities.

Perhaps the greatest difficulty in setting up these programmes is to attract and retain capable nursing and medical staff in the paediatric ICU.

\section{Jamaica}

Chain of Hope was approached in 2001 by the Bustamante Hospital for Children, Kingston, Jamaica, to assist them with the treatment of complex cardiac lesions. In total, 400 children are born on the island each year with $\mathrm{CHD}$, and over half require intervention. Rheumatic heart disease also poses a problem. Bustamante Hospital for Children is the only paediatric hospital in Jamaica and the only specialist paediatric hospital serving the Englishspeaking Caribbean. Bustamante has five paediatric intensive care beds, and these are the only publicly funded paediatric ICU beds in the entire island, which serves a population of 2.7 million. Cardiac cases are performed on an emergency basis and when elective cases are able to compete with general paediatric ICU cases. Therefore, most of the paediatric cardiac surgical cases are conducted through international visiting missions' teams such as Chain of Hope, Congenital Heart Institute of Florida, and Caribbean Heart Menders. Each mission conducts 10-15 surgeries and a similar number of catheterbased interventions. The catheterisations are conducted at the University Hospital of the West Indies because this is the only cardiac catheterisation lab on the island. The missions sent by Chain of Hope in the early years consisted of up to 25 volunteers. However, this has reduced significantly since Chain of Hope was asked to help develop the local team's skills. Chain of Hope funded and organised the training of two cardiologists for Bustamante, one cardiac anaesthetist-intensivist, and a cohort of 45 cardiac nurses. Together with a partner charity in this project, Gift of Life International, the first perfusionist employed to Bustamante Hospital was trained overseas and is now back on the island. Training has been both onsite and overseas, and a vital component is the hands-on experience. The mission teams provide extra mentorship, but the training conducted has been mainly outside of missions - e.g., bringing local clinicians to the United Kingdom for training. It can be argued that the larger the mission team, the less opportunity for the local team to really learn. Consequently, Chain of Hope has measured its success over the years by the reduction in mission personnel. The main obstacle to a sustainable paediatric service is the lack of paediatric ICU beds at Bustamante. Therefore, Chain of Hope has led a group of stakeholders to fund a new cardiac wing at the Bustamante Hospital for Children, which will include a cardiac catheterisation laboratory, a dedicated cardiac operating theatre, and five dedicated cardiac intensive care beds with a high dependency unit. The future for the paediatric cardiac service will depend on the ability of the new cardiac wing to remain a dedicated cardiac unit, which will require its own revenue stream to support its independence. In May, 2017, Chain of Hope funded a manager to run the cardiac wing as a separate specialist service to the hospital. The aim is to start with 150 annual operations and increase this by 50 the following year. Missions will be reduced to 2-6 clinicians so that the incoming teams are supporting the locally trained team to conduct their service on a regular basis. The optimal solution is to employ nurses and intensivists to be based in Jamaica to support the local team.

\section{Conclusions}

We present four different models of successful partnership projects that have established sustainable paediatric cardiac surgical and cardiac intensive care programmes in low- and middle-income countries. The Mexico team created a hybrid programme unifying the efforts and resources from a public and a private hospital with the help of a not-for-profit foundation, and a twinning programme with an academic paediatric hospital in a high-income country. Continuous support from fundraising efforts from Kardias and continuous support from Texas Children's Hospital via telemedicine and regular visits to the local team are key to the sustainability of this growing and successful programme in Mexico. The centres in India and Vietnam have improved patient outcomes and built sustainable programmes through education of nursing and medical staff and creating alliances with multidisciplinary capacity-building non-governmental organisations. Chain of Hope has helped the team in Jamaica by focusing their efforts on the training of the local surgical, anaesthesia, perfusion, and intensive care teams. They thus build up the 

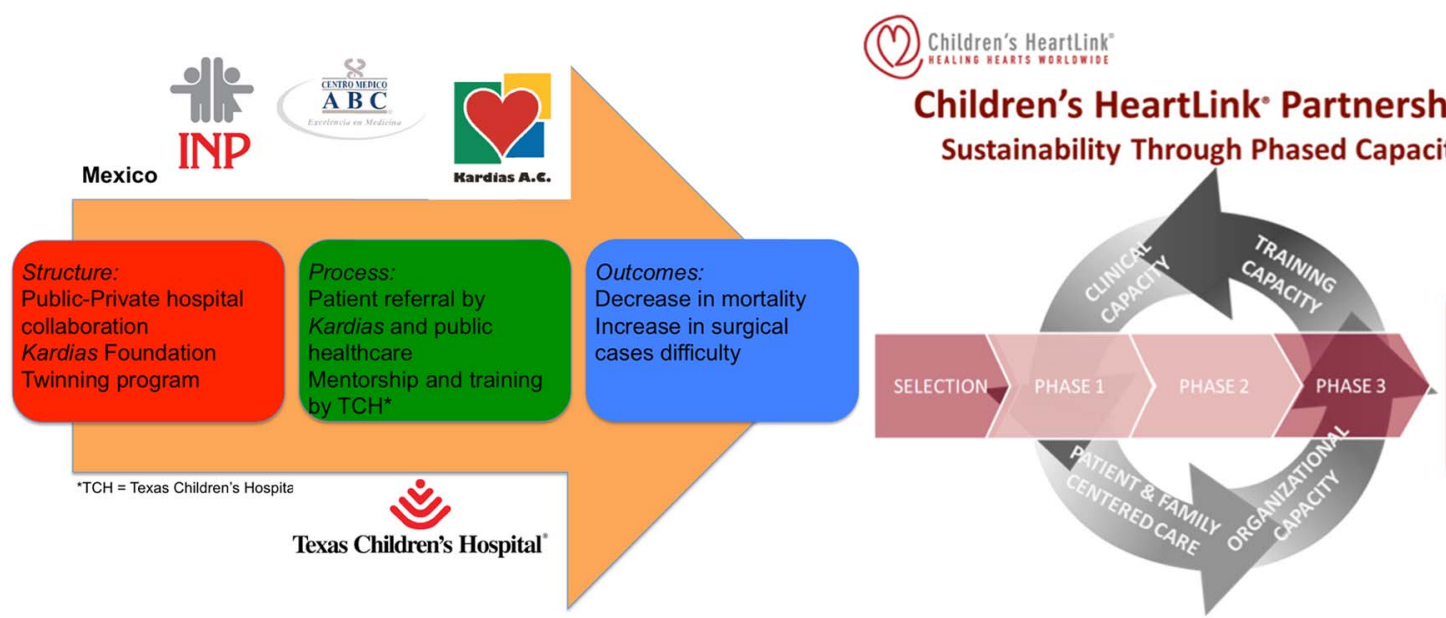

Children's HeartLink Center of Excellence

Figure 2.

The Mexico (on the left) and Asia (on the right) projects represented by the Donabedian model of healthcare assessment. Structure refers to the context in which care is delivered; process refers to the transactions between patients and providers, and outcomes describes the effects of healthcare on the health status of those patients.

local team's autonomy, which is fundamental for longterm sustainability of their paediatric cardiology and cardiac intensive care programme.

Figure 2 shows our programmes in Mexico and Asia based on the Donabedian model. The Donabedian model is a valuable means of assessing the quality of healthcare. It uses information drawn from three categories: structure, referring to the context in which care is delivered; process, related to transactions between patients and providers; and outcomes, describing the effects of healthcare on the health status of those patients.

Paediatric cardiac surgery and cardiac intensive care services in low- and middle-income countries remain very limited. Common barriers to achieving comparable outcomes to high-income countries go beyond limited economic resources. Multidisciplinary team training, continuous support via telemedicine or easily accessible media, and building alliances with nonprofit organisations that help with quality improvement initiatives, on-going training and building self-sufficient, fully integrated paediatric cardiac programmes are fundamental components to establish sustainable partnership programmes in paediatric cardiac surgical and cardiac intensive care programmes in low- to middle-income countries.

\section{Acknowledgements}

The authors thank all team members in these multicentre programmes for their outstanding dedication to improve the outcomes of all children with heart disease.

\section{Financial Support}

This research received no specific grant from any funding agency, commercial or not-for-profit sectors.

\section{Conflicts of Interest}

None.

\section{References}

1. Nguyen N, Leon-Wyss J, Iyer KS, et al. Paediatric cardiac surgery in low-income and middle-income countries: a continuing challenge. Arch Dis Child 2015; 100: 1156-1159.

2. The International Surgical Outcomes Study Group. Global patient outcomes after elective surgery: prospective cohort study in 27 low-, middle- and high-income countries. Br J Anaesth 2016; 117: 601-609.

3. Corno AF. Paediatric and congenital cardiac surgery in emerging economies: surgical 'safari' versus educational programmes. Interact Cardiovasc Thorac Surg 2016; 23: 163-16.

4. Khan A, Abdullah A, Ahmad H, et al. Impact of international quality improvement collaborative on congenital heart surgery in Pakistan. Heart 2017; 103: 1680-1686.

5. Hoffman JI. The global burden of congenital heart disease. Cardiovasc J Afr 2013; 24: 141-145.

6. Staveski SL, Zhelva B, Paul R, et al. Pediatric cardiac surgery Parent Education Discharge Instruction (PEDI) program: a pilot study. World J Pediatr Congenit Heart Surg 2015; 6: $18-25$.

7. Staveski SL, Parveen VP, Madathil SB, Kools S, Franck LS. Parent education discharge instruction program for care of children at home after cardiac surgery in Southern India. Cardiol Young 2016; 26: 1213-1220.

8. Staveski SL, Parveen VP, Madathil SB, Kools S, Franck LS. Nurse and parent perceptions associated with the Parent Education Discharge Instruction Programme in southern India. Cardiol Young 2016; 26: 1168-1175.

9. Dearani J, Neirotti R, Kohnke E, et al. Improving pediatric cardiac surgical care in developing countries: matching resources to needs. Semin Thorac Cardiovasc Surg Pediatr Card Surg Annu 2010; 13: $35-43$.

10. Balachandran R, Kappanayil M, Sen AC, et al. Impact of the international quality improvement collaborative on outcomes after congenital heart surgery: a single center experience in a developing economy. Ann Card Anaesth 2015; 18: 52-57.

11. Jenkins K, Castañeda A, Cherian K, et al. Reducing mortality and infections after congenital heart surgery in the developing world. Pediatrics 2014; 134: e1422-e1430. 